
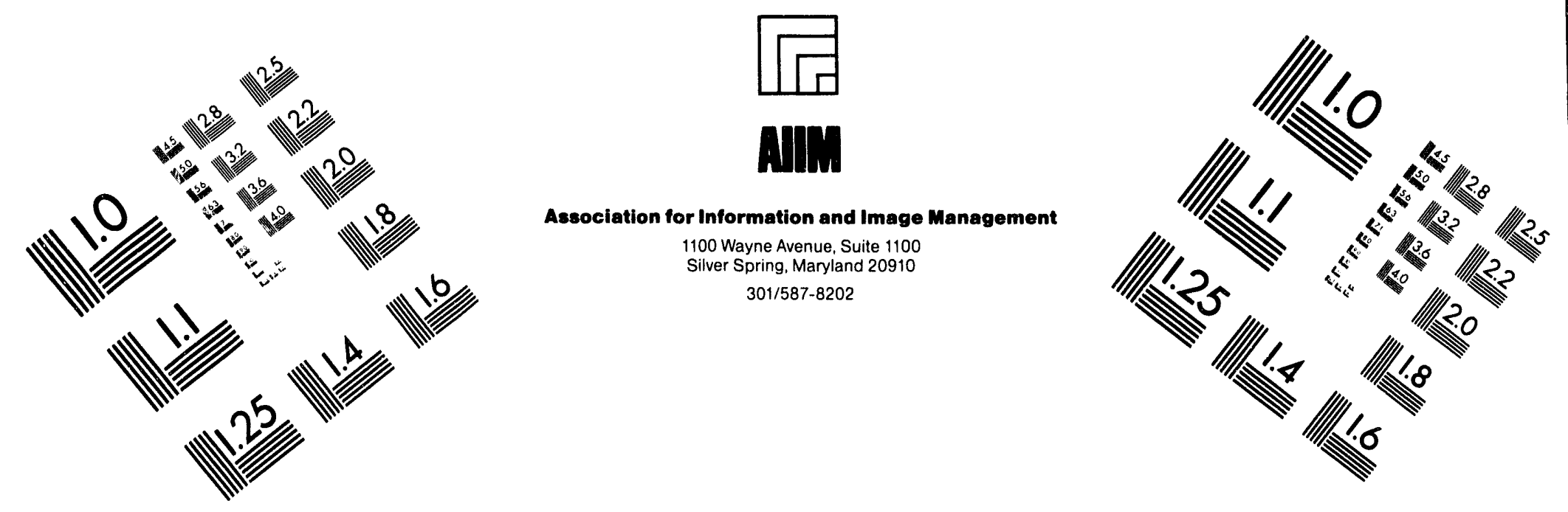

\title{
Centimeter
}

$\begin{array}{llllllllllllllll}1 & 2 & 3 & 4 & 5 & 6 & 7 & 8 & 9 & 10 & 11 & 12 & 13 & 14 & 15 & \mathrm{~mm}\end{array}$

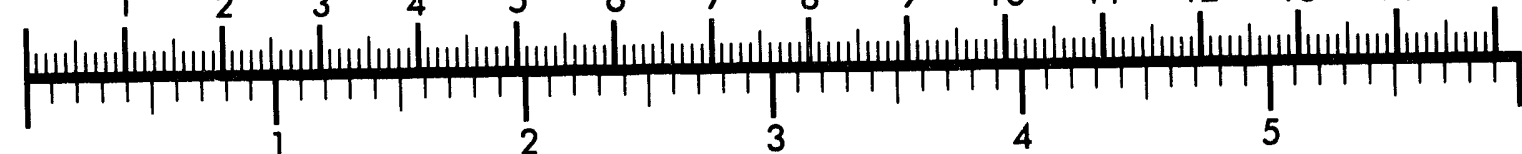

Inches
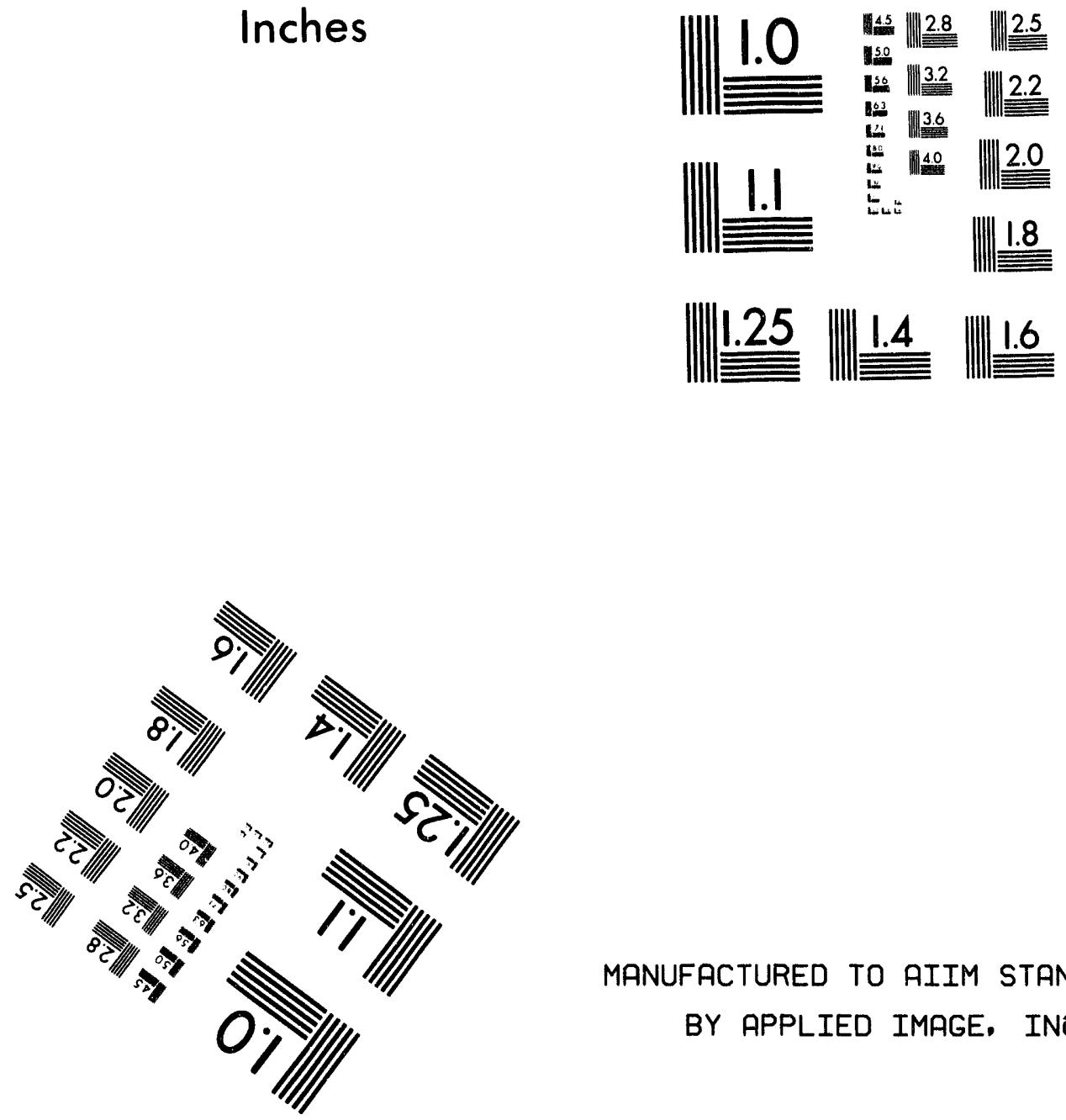

MANUFACTURED TO AIIM STANDARDS BY APPLIED IMAGE. INC.

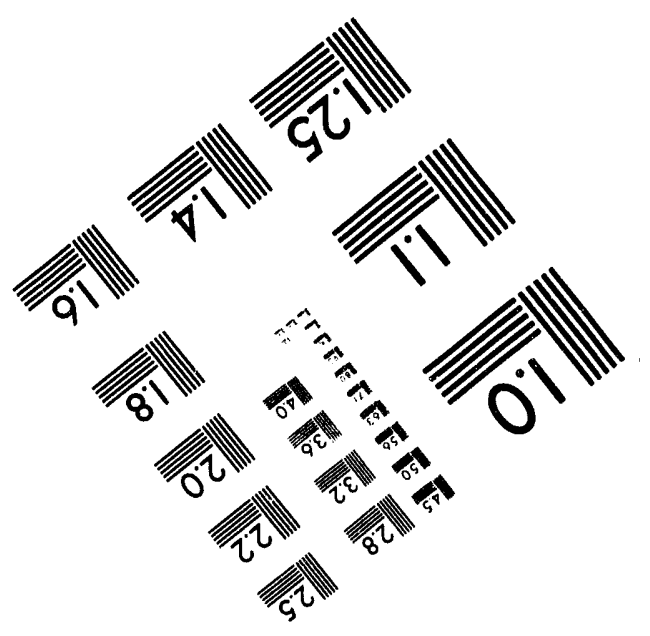



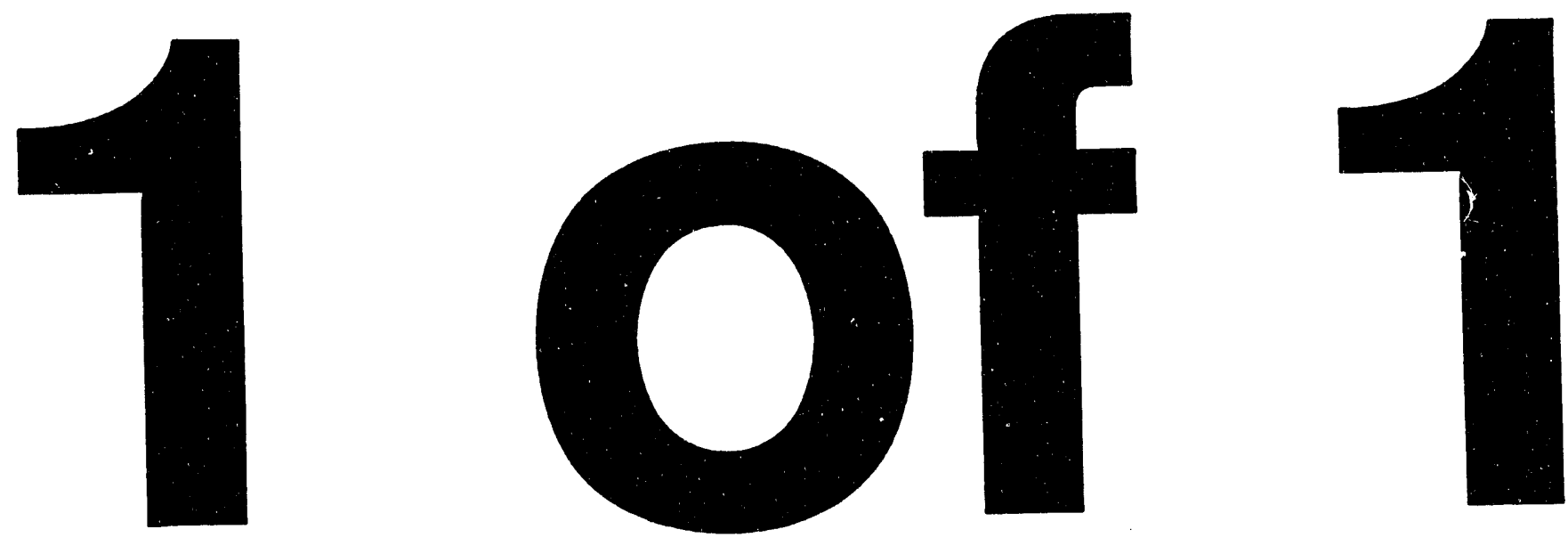
SAND94-0514

Distribution

Unlimited Distribution

Category UC -510

Printed June 1994

\section{Defining a Controller Architecture for the Long-Reach Manipulator}

William E. Ford

Intelligent Systems Department III

Sandia National Laboratories

Albuquerque, New Mexico 87185

\section{Abstract}

Which type of robot controller should be used on the Long-Reach Manipulator? This report defines various approaches, discusses the advantages and disadvantages of each, and describes a recommended approach. Knowledge from past procurements and new technology development is included in this analysis to form the basis for a n.u urement specification for this robotic system.

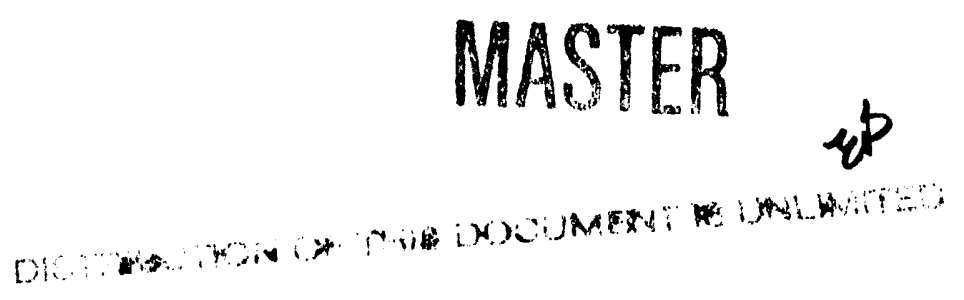




\section{Acknowledgments}

The author thanks David J. Miller for the use of his paper on "Standards and Guidelines for Intelligent Robotic Architectures" (Proc of AIAA Space Programs and Technologies Conference Exhibit, September 21-23, 1993). The information served as the basis for this report. 


\section{Contents}

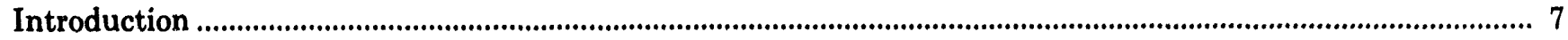

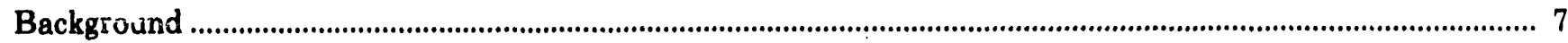

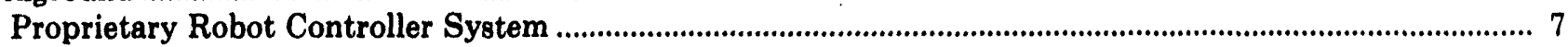

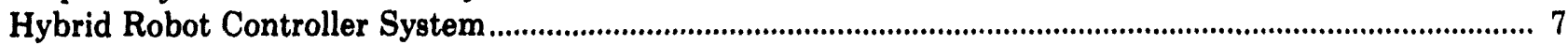

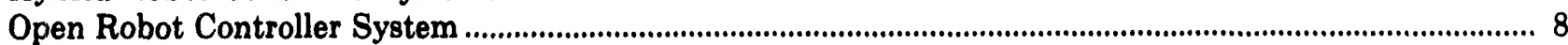

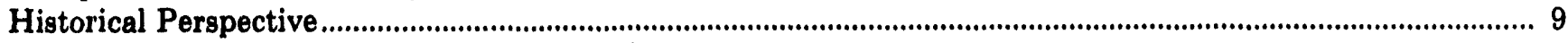

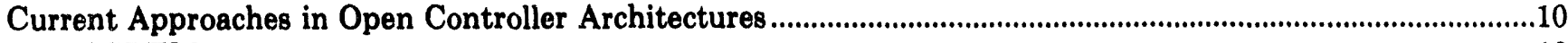

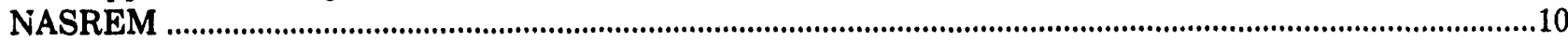

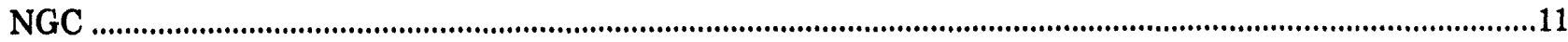

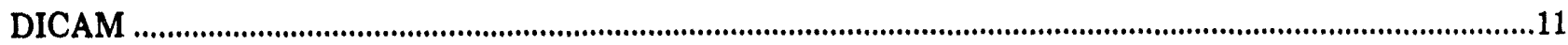

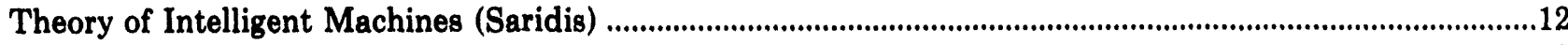

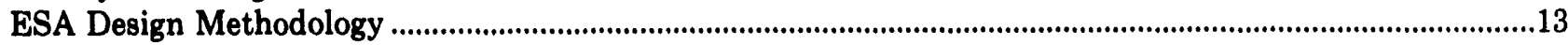

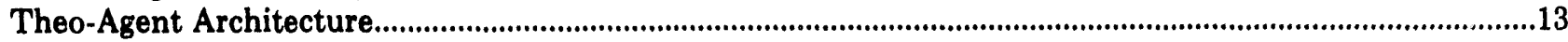

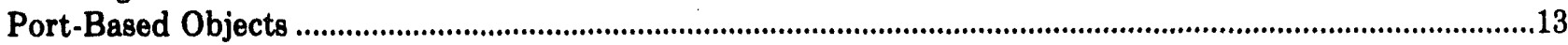

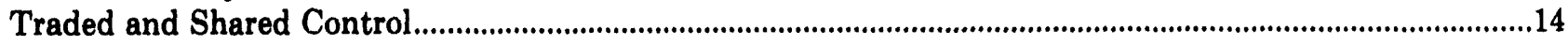

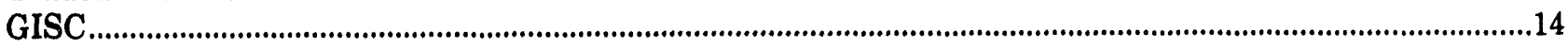

Telerobotic Architecture for Large Aircraft Maintenance ......................................................................15

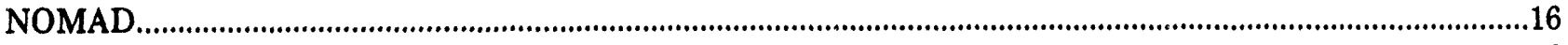

ROBLINE

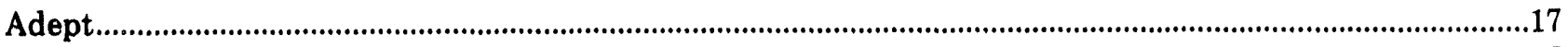

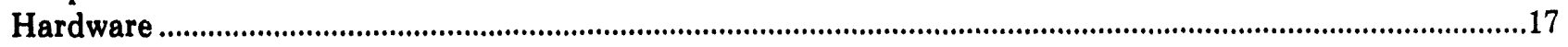

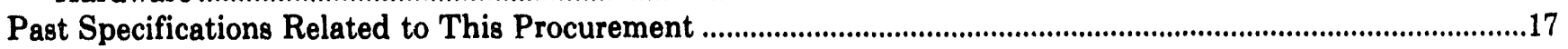

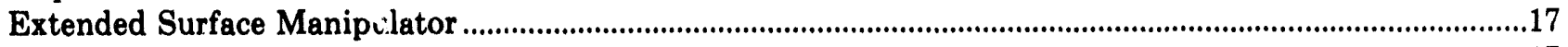

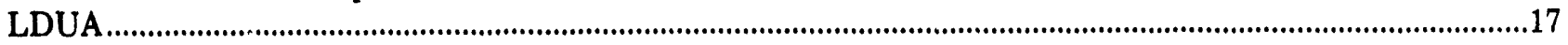

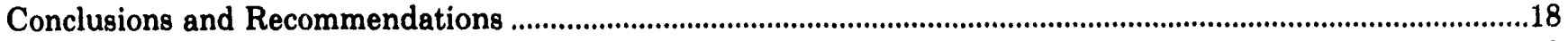

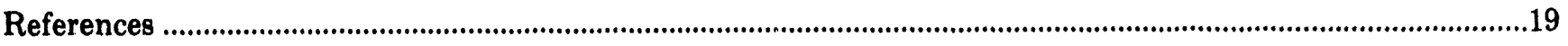

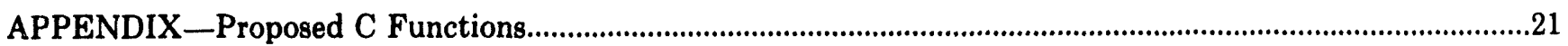

\section{Figures}

1 A Heirarchical Control System Architecture for Telerobots ...................................................................10

2 Individual Controller Reference Architecture: Domain and Meta-Control ..................................................12

3 Three-Level Intelligent Machines Architecture .......................................................................................13

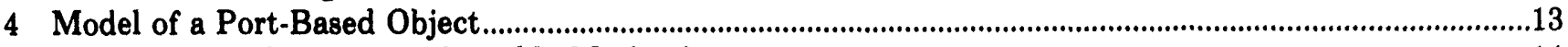

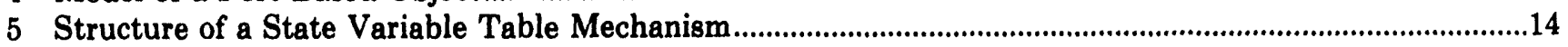

6 Generic Intelligent System Controller (GISC) Architecture ...............................................................14

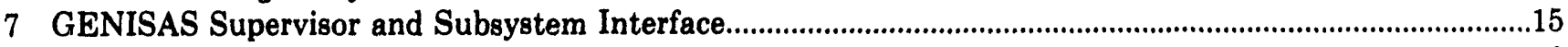

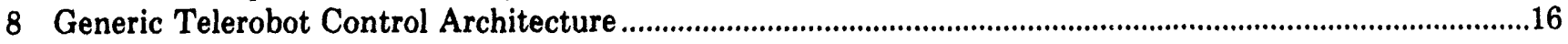




\section{Defining a Controller Architecture for the Long-Reach Manipulator}

\section{Introduction}

To draft a procurement specification for the Long-Reach Manipulator (LRM), we first need to determine the benefits and limitations of the various robotic control system architectures available to us. This report identifies and describes the advantages and potential disadvantages of using an open control system versus a closed (or proprietary) system, focusing on integration of interfaces for sensors, end effectors, tooling, and operator interfaces. In addition, the various controls methodologies of several recent systems is described. Finally, the reasons behind the recommendation to procure an open control system are discussed.

\section{Background}

We classify robot controllers into three categories: proprietary (also called "closed") systems, hybrid systems and open systems:

- A proprietary system limits access for integrating external hardware (including sensors and software).

- With a hybrid system, certain aspects (such as the control laws) are closed whereas most other elements are accessible.

- The hardware and software structure of an open system allows us to easily modify all aspects of the design (e.g., sensor integration, operator interfaces such as force reflection, and new control laws for servos) without consulting the original manufacturer.

Most of the robotic systems sold throughout the world today contain proprietary controllers, which is desirable if the application is well defined and is not expected to change. This type of "turn-key" system requires little in-house expertise and development. Recently, however, the demand for more agility has dramatically increased the use of controllers with open architectures. The following describes some of the advantages ana disadvantages of each of the three robot controller categories as they apply to the LRM specification.

\section{Proprietary Robot Controller System}

The advantages and disadvantages of a proprietary robot controller are discussed in this section.

\section{Advantages of a Proprietary System}

Major reasons to select a proprietary robot controller are its large installed user base (because of its proven track record) and its low cost (because of the amortization of the robot controller development over a large number of units). If a customer is highly confident in knowing the current and future tasks of the robotic system, then his preferred choice is a proprietary system that meets his needs.

\section{Disadvantages of a Proprietary System}

What happens if a proprietary system that meets the customer's needs does not exist and customization is required? Proprietary systems do not readily leverage off of other controller capabilities; thus, any enhancements required for the closed architecture will need to be internally developed. Only the original developer can modify a proprietary system, and this can be expensive or technically impossible without extensive redesign.

Even trying to rapidly field a new robot requires an excessive amount of time. As the world exists today, most robot manufactures have their own proprietary control system. This does not facilitate cooperative team-based robotics system development nor promote common approaches to robot control.

\section{Hybrid Robot Controller System}

The following describes the advantages and disadvantages of a hybrid robot controller.

\section{Advantages of a Hybrid System}

Some of the benefits of a proprietary controller, with the ability to customize and upgrade the system 
as needed, can be obtained with a hybrid system. With this approach, the vendor provides the low-level control functions and a way to externally access these functions. For example, the vendor might be responsible for servoing joint angles that are generated by external software at a given update rate (typically 10 to $250 \mathrm{~Hz}$ ). Thus, this proprietary part of the system could interact with the trajectory generation, sensor integration, and operator interface systems, which could all be based on an open architecture.

A few of the results of a hybrid system that we are seeing today are:

- The integration of advanced subsystem modules as they are developed-without having to redevelop all of the system components.

- Faster technology development from the adaptation of previously developed generic technology to new applications.

- Safer robotic systems because of the reuse of modular generic technologies in diverse applications.

We can characterize robotic systems that reuse well-understood generic technologies much better than robotic systems that use technology developed only for a particular application. When the development satisfies the needs of many applications, the total life cycle cost and time for technology development are reduced, because effort is not duplicated. Also, technology that is generally applicable results in cheaper final systems, because development costs can be amortized over many systems.

The U.S. Department of Energy's (DOE) Robotics Technology Development Program (RTDP) has used the hybrid approach to develop faster, safer, and cheaper robotics technologies for hazardous waste site cleanup-an unstructured environment with evolving needs. The DOE has employed generic technologies that allow the creation of robotic systems from welltested subsystem modules, including commercially supplied items such as low-level robotic controllers. ${ }^{1}$ In the future, generic technology will have applications outside of hazardous environments, which will reduce the costs of commercial technology. Reuse of familiar technology will also reduce operator training time.

Hybrid and open systems share similar advantages. For more information, refer to the section titled Open Robot Controller System.

\section{Disadvantages of a Hybrid System}

To perform today's complicated tasks, intelligent robotic systems are pushing the limits of existing technology, and the cost of implementing the new, complex technology is rapidly increasing. In the past, much time was spent developing and redeveloping the communication and subsystem interface infrastructure. Thus, the ability to add high-level intelligence to today's systems is often hindered by the initial system infrastructure development efforts. ${ }^{2}$ A system software architecture should bridge the gap between high-level subtask descriptions and low-level robot commands. Current commercial robot control systems fail to bridge this gap, and customized architectures that allow higher intelligence to be added are expensive, require significant time and personnel, and are rarely adaptable to new applications. Open architectures that are easily adaptable to new applications are needed.

Suppose a developer was forced to use an application program interface dictated by the manufacturer. This would preclude adding greater functionality at the low-level control; the control laws and servo gains would be inaccessible. A third party could offer improved performance if these parameters could be changed or modified.

Only an open system is able to quickly integrate new sensors while rapidly and efficiently modifying existing software structures. An open system allows us to get the right data to the right place in an appropriate, timely fashion, and to implement newer and potentially more complex control laws that take advantage of the new data.

\section{Open Robot Controller System}

This section discusses the advantages and disadvantages of an open robot controller.

\section{Advantages of an Open System}

An open architecture simplifies interfacing to nonstandard subsystems. This type of communications-based system structure also promotes flexibility, which in the past has been difficult to achieve because of the immense integration difficulties associated with multivendor equipment and hardware/ software interfaces. ${ }^{3}$ As with a hybrid system, an open architecture utilizes the following concepts:

- A controller based on a nonproprietary computer platform (e.g., Sun, SüI, PCs).

- A standard operating system (e.g., UNIX) and a standard control language (e.g., $\mathrm{C}$ or $\mathrm{C}++$ ).

- Hardware based on a standard bus architecture (e.g., VME) that can interface with a variety of peripherals and sensor devices.

- A communication scheme (e.g., a network) that 
allows workcell controllers to share databases and to operate from remote locations.

One of the greatest benefits of an open architecture approach for any key technology is the ability to foster competition. A user company can add value to the hardware or software, and this long-term gain allows a system to be continually improved to meet unanticipated or changing needs, overcoming the immediate gains offered by selecting a proprietary system. By enabling robot controller retrofits to be provided on a quality and needs basis, an open approach to hardware and software implementation can stimulate competition among vendors of subsystem technology. A common approach will stimulate the robot industry to grow, with third-party companies developing hardware and software packages that can lower implementation costs through competitive, multiple-source procurements rather than relying only on the original system developers. Competition also fosters rapid conversion from research systems to operational systems, thus shortening the research-to-commercial product cycle.

Another great benefit of open (and hybrid) controllers is the reuse of elements of an existing system: ${ }^{2}$

-When we reuse existing capabilities that contain tested, well-characterized components, we know that they will meet our requirements for quality and safety and not fail catastrophically in unexpected ways.

- We can take advantage of rapid improvements in cost and performance driven by high-volume markets.

- Open and hybrid architecture robot controllers based on modular, distributed, interconnected, generic components allow leveraging of future advances in hardware and software for improved throughput, higher precision, and greater intelligence.

- With open controller designs, a customer can standardize on platforms, operating systems, and user interfaces.

- Common open controller technologies promise reductions in training requirements, system support requirements, and maintenance costs.

- Programming is simplified and development time and cost are saved because of prior experience with the system.

To summarize, open architecture controllers provide greatly improved flexibility, reconfigurability, extendibility, interoperability, interchangeability, portability, scalability, reliability, and reusability.

\section{Disadvantages of an Open System}

We can see some potential problems with an open or flexible system:

- Initial stimulation of the market will be expensive, and we will have to pay manufacturers to implement open system principles.

- Initially, only the larger robot manufacturers will offer a phased approach to open systems. That is, only a few of the Application Programming Interfaces (APIs) will be published, giving the consumer few choices. Years from now, however, the consumer may be overwhelmed by the number of choices. What would the criteria be for making a decision?

- Unlimited flexibility may lead to overly complex systems.

\section{Historical Perspective}

Two National Institute of Standards and Technology (NIST)/DOE workshops were held in January 1990 and January 1991 to determine what generalized approaches should be employed in the development of robotic control systems for DOE hazardous waste cleanup operations. The goals of the workshops were to:

- Identify a system concept that would stimulate cooperative team-based robotics system development.

- Facilitate the accelerated fielding of integrated robot system demonstrations.

- Stimulate common approaches to robot system control. ${ }^{4}$

The first workshop concluded that adopting existing architectures was inappropriate; instead, DOE should (1) focus on problem solving (rather than on infrastructures), (2) stress the reduction of implementation costs, and (3) strive toward modular open systems by stimulating subsystem integration. ${ }^{5}$ The second workshop concluded that DOE researchers should (1) continue to stress modularity; (2) use RS232, RS422, or Ethernet for communications; (3) use VME computer hardware, VxWorks for the real time operating system, and $\mathrm{C}$ and $\mathrm{C}++$ for the software implementation where possible; and (4) stress object-oriented techniques. ${ }^{6}$ In addition, the importance of distributed multiprocessing computing environments for complex system control was stressed.

All of the above conclusions supported a generic approach. As a result of the two workshops, the DOE 
EM-50 program office began the RTDP thrust into the development of a generic system architecture - the Generic Intelligent System Controller (GISC) approach-which is discussed in the next section. Approximately 2 years later, six RTDP projects were reviewed for their GISC compatibility. ${ }^{7}$ The consensus was that the projects displayed various levels of GISC compatibility and are consistent with the overall GISC philosophy.

\section{Current Approaches in Open Controller Architectures}

This section summarizes some of the most recent approaches to developing intelligent robotic systems. It is not meant to be all-encompassing but to be a survey of several current approaches.

\section{NASREM}

The National Aeronautics and Space Administration/National Bureau of Standards (NASA/NBS)
Standard Reference Model for Telerobot Control System Architecture, NASREM, is based on research from several NASA, NIST, DOE, and university research centers. This approach was first implemented on the Space Station Flight Telerobotic Servicer. ${ }^{3}$ It is one of the first attempts to fully specify all the components of an intelligent system. ${ }^{2}$

The NASREM Standard Reference Model telerobot system architecture is a conceptual model that decomposes into a three-legged hierarchy of levels (Figure 1). ${ }^{8}$ Each of these levels performs a different fundamental mathematical transform. The architecture also splits each level into three sections: task decomposition, world modeling, and sensory processing. Thus, every level is composed of three modules. Each module is a finite-state machine that accepts inputs, computes some function based on state and inputs, and produces outputs. The inputs consist of commands from higher levels, sensory data from the same level, and status/data from lower levels. The outputs are commands for the lower levels, processed sensory data for the same level, and data/status feedback for upper levels.

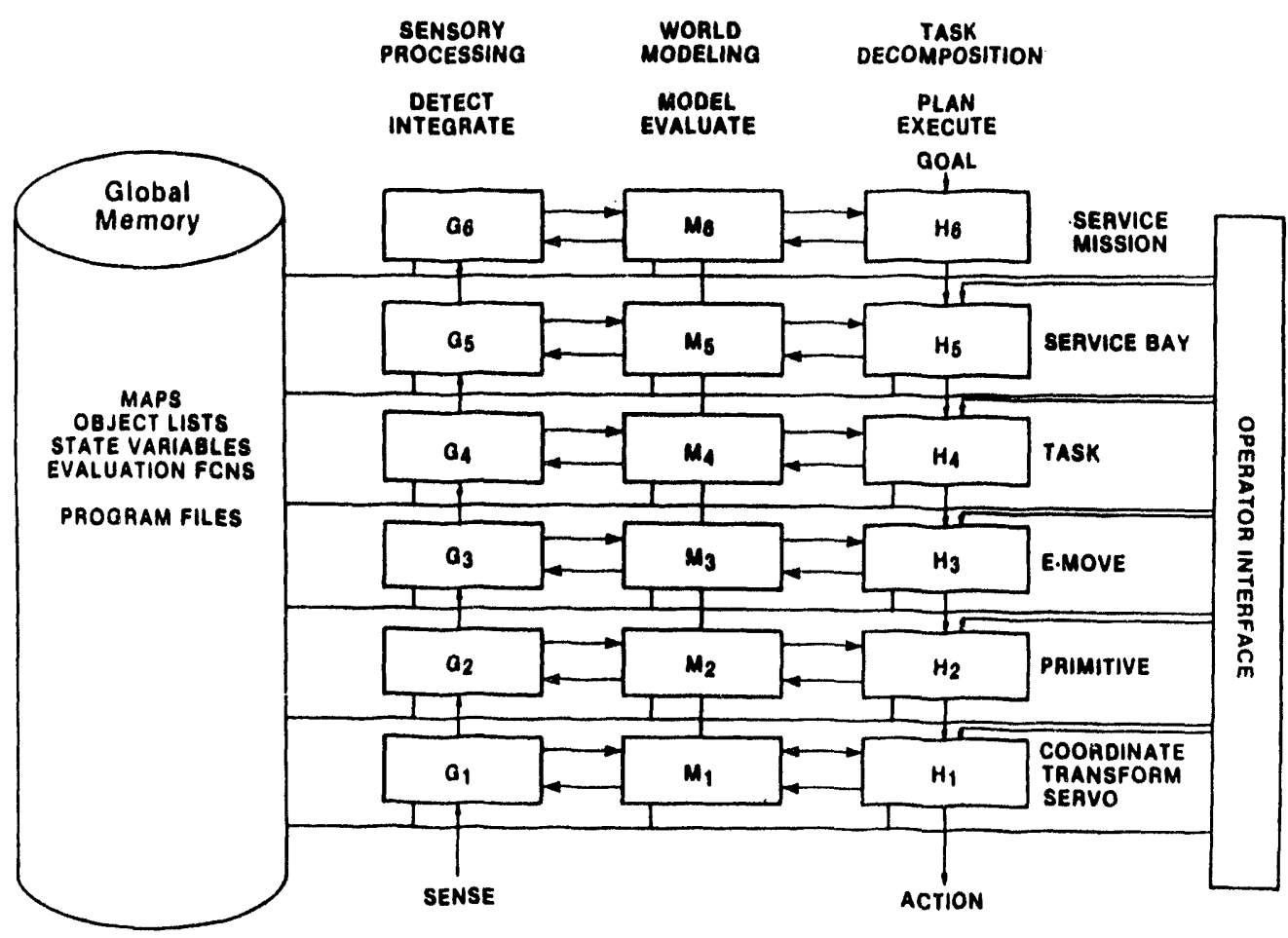

Figure 1. A Hierarchical Control System Architecture for Telerobots (J. S. Albus et al, ref. 8). 
The information provided in ref. 8 is only a standard methodology for implementing large telerobot control systems and not a physical working system. It is the author's opinion that NASREM's fine-grained decomposition of tasks forces an application-dependent software structure. ${ }^{2}$ Its multilevel organization of finite-state machines creates difficulties for independent software developers, because the higher levels of the NASREM hierarchy have never been elaborated and the lower levels cannot isolate machine-dependent parts of the hardware interfaces. The problem is further complicated by the use of a large global memory that does not support the basic principles of encapsulation and information hiding. Furthermore, the common memory creates a large, uncontrolled interface with maintenance problems. ${ }^{2}$

\section{NGC}

The Next Generation Controller (NGC) is an attempt to develop a specification for an open system architecture standard (SOSAS) that will become an American National Standards Institute standard for machine tools. ${ }^{9}$ Sponsored by the U.S. Air Force, this project is important, because it will provide welldefined standards for the loosely specified NASREM concept. Martin Marietta Corp. was chosen as the prime contractor for the NGC program. A total of 27 voting and 350 nonvoting members make up the three boards or committees on the project. ${ }^{3}$

The objectives of the specification are to define an open architecture that provides increased capability, flexibility, and reduced cost/performance ratios for workstation/machine controllers. The approach requires all modules to be "plug" compatible between different manufacturers. The NGC is based on a virtual machine model in which each module has publishad specifications for its functionality and interfaces. This enables others to independently develop and test components that will extend or modify the capabilities of a controller without resorting to controller redesign. Three concepts are contained within the framework:

- An integration architecture that provides a set of standard services for application programs.

- A set of four applications that supplies a basic set of machine-controller functionality with message-passing facilities called the Neutral Manufacturing Language.

- An integration/configuration environment that provides a standard set of tools for augmenting, modifying, and integrating modules into a congruous system.
Because prototype systems using the NGC approach are currently in the early stages of development, it is premature to judge the success or failure of the effort. Version I of the SOSAS specification encompasses six volumes totaling over 1,000 pages; therefore, most vendors will probably require substantial time and effort to become proficient in applying NGC standards to system control. ${ }^{2}$

Because version I of the specification is too complex, the National Center for Manufacturing Sciences (NCMS), Martin Marietta Corp., and the University of Texas at Arlington are currently under contract to redefine SOSAS. ${ }^{10}$ The new document will be based on twin foundations of standards references for the hardware/software platform and on object-oriented system analysis for application software. The applications are replaced by a component-level architecture expressed in terms of responsibilities, requirements (inputs), and products (outputs). The primary architecture is a reference architecture; that is, it contains no taxonomy - it is simply a pool of primitive responsibilities (with constraints) that may be organized into an application architecture. Interoperability is described at the source code level. Prototype systems using the NGC approach are currently in the early stages of development.

\section{DICAM}

CimFlex Teknowledge Corp. is undertaking a 4-year effort, which began in 1992, to develop a new technology foundation and associated methodology for the rapid development of high-performance intelligent controllers. These controllers will be employed in distributed intelligent control and management (DICAM) applications. This research is part of the Advanced Research Projects Agency's DomainSpecific Software Architecture (DSSA) initiative. ${ }^{11}$ The company is attempting to fuse the best ideas from knowledge engineering and software engineering into the intelligent real-time control software being developed for an application and into the software development process itself. This generic control architecture combines a task-oriented domain controller with a meta-controller that schedules activities within a domain controller (Figure 2). The project has four elements:

- The formulation of a reference architecture for intelligent control.

- The construction of applications in a development workspace. In this workspace, system requirements are ultimately satisfied by choosing design components that specialize and particularize components of the generic reference architecture. 


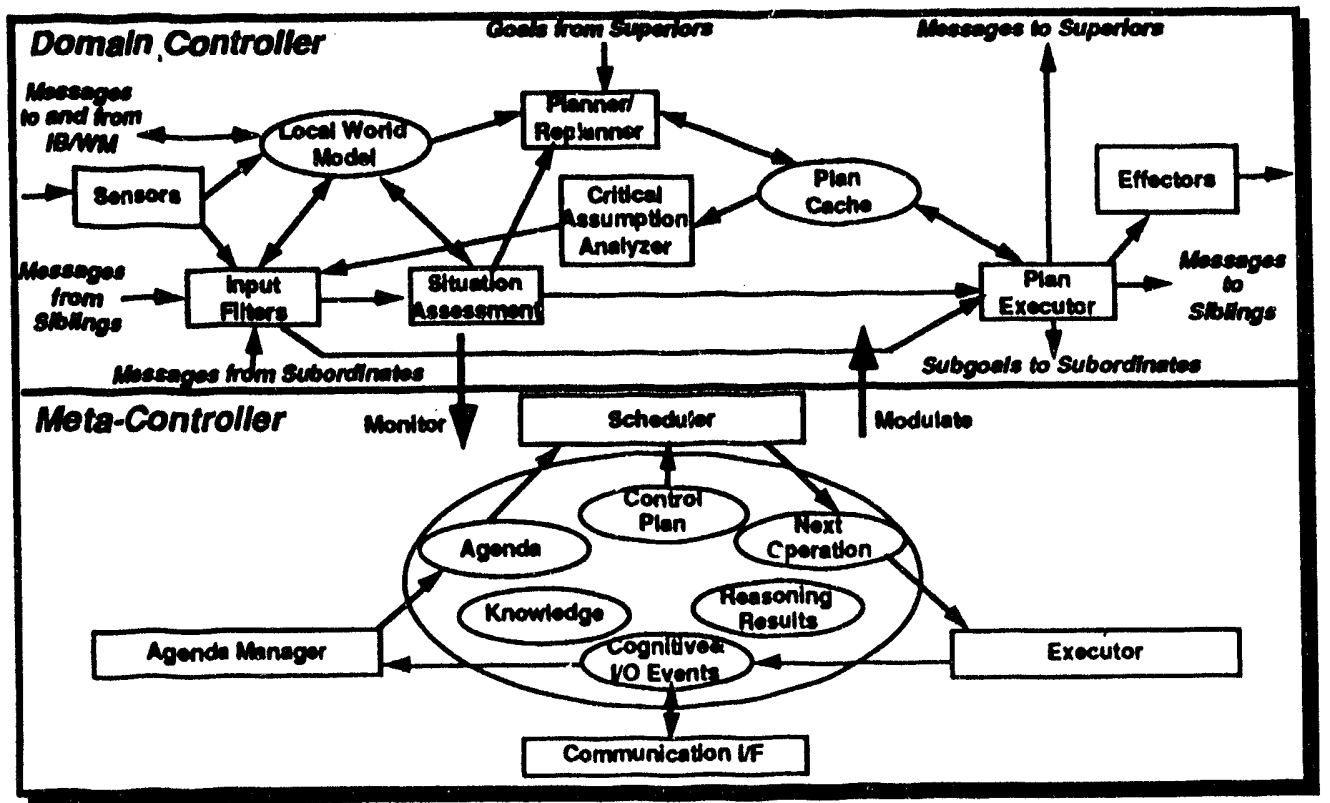

Figure 2. Individual Controller Reference Architecture: Domain and Meta-Control (F. Hayes-Roth et al, ref. 11).

- The construction of reusable modules for an application-building repository.

- The creation of a rich array of development tools that incorporate numerous techniques from both software engineering (e.g., control law specifies, code generators, protocols, compilers, and debuggers) and knowledge engineering (e.g., domain modelers, requirements managers, and knowledge-based design assistants).

CimFlex Teknowledge Corp. demonstrated the first working prototype of their development environment in August 1992. They claimed that the demonstration showed how requirements can constrain and direct the software development process to produce software that specializes the generic controller reference architecture. ${ }^{12}$ Since this approach is currently in the early stages of development, it is premature to judge the success or failure of the effort.

\section{Theory of Intelligent Machines}

Professor George Saridis of the Rensselaer Polytechnic Institute has been advancing an Intelligent Machines architecture since $1979 .{ }^{13}$ Intelligent machine functionality is implemented by intelligent controls using entropy as the metric. This approach is based upon a hierarchical control structure of three levels (Figure 3):
- First, the organization level is modeled after a Boltzmann machine for abstract reasoning, task planning, and decision making.

- Second, the coordination level is composed of a structure of Petri net transducers acting as coordinators that are supervised by a dispatcher. The dispatcher also interfaces with the organization level.

- Third, the execution level consists of the sensory and motion-control hardware that interacts one-to-one with the coordinators above.

The entire architecture is based on the principle of increasing precision with decreasing intelligence. This means that the highest level tasks are assigned to the highest machine intelligence level and smallest complexity (database size), and the lowest level tasks are assigned to the lowest machine intelligence level and largest complexity.

The system is currently implemented on a robotic transporter designed for space construction. Professor Saridis claims that, compared to other proposed architectures, the resulting software structure is extremely efficient, effective, versatile, and capable for remote operation. This theory is still being tested, and it will be interesting to see any successes or failures of the implementation phase. 


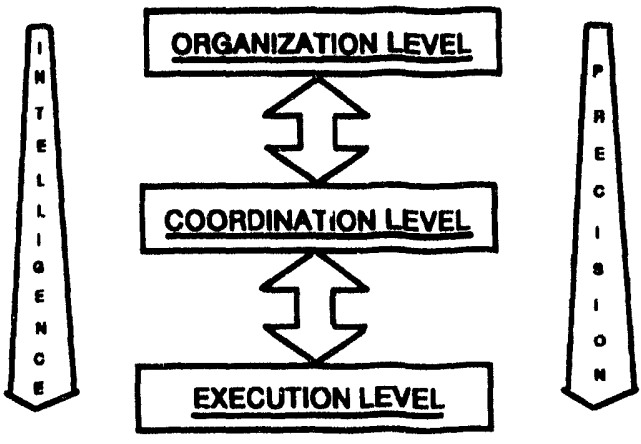

Three-Level Structure

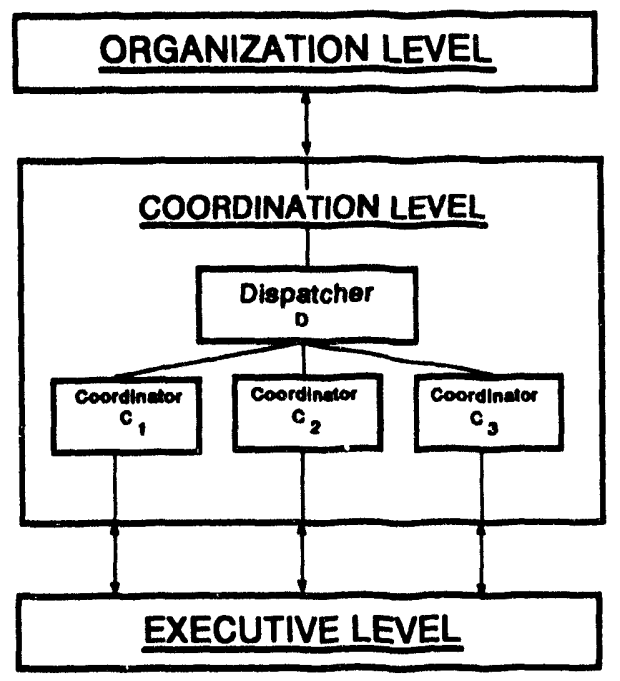

Topology of the Coordination Level

Figure 3. Three-Level Intelligent Machines Architecture (G. N. Saridis, ref. 13).

\section{ESA Design Methodology}

The European Space Agency is establishing a design methodology for space and automation programs that is based on logical reference models. ${ }^{14}$ The goal is to be able to clearly trace requirements to design solutions so that technical decisions can be justified. This approach should avoid the complicated flexible solution that requires high technical risk and huge development cost. Three logical models are defined:

- A functional reference model, which represents the decomposition of functions and information flow.

- An application reference model, which represents specific classes of applications.

- An operations reference model to focus on modes of operation/interfacing.

ESA does not define an architecture. It provides a procedure by which we can consider architectures generically while concentrating on system requirements and formulating a minimal cost-effective solution. ${ }^{2}$

\section{Theo-Agent Architecture}

Carnegie-Mellon University has developed a Theo-Agent architecture that combines a stimulusresponse subsystem for rapid reaction with a searchbased planner for handling unanticipated situations. ${ }^{15}$ A robot agent continually chooses which action to perform using the stimulus-response subsystem when possible and falling back on the planning subsystem only when necessary. Whenever the agent must plan, it applies an explanation-based learning mechanism to formulate a new stimulusresponse rule that covers the new situation and others similar to it. With experience, the agent acquires new stimulus-response rules that eliminate the need to plan; thus, the agent becomes increasingly reactive. The architecture was implemented on a Hero 2000 robot that was used to locate garbage cans. The author listed several limitations, the most significant being the scalabilty issue and the appropriate algorithm choice.

\section{Port-Based Objects}

Another Carnegie-Mellon University development has produced a software framework based on dynamically reconfiguring software for sensor-based control systems. ${ }^{16,17}$ The approach combines objectoriented design with port-automaton design of digital control systems. Thus, a port-based object is defined as an object that has various ports for communication (Figure 4). The internal states and methods are hidden from other objects-only the ports of an object are visible to other objects.

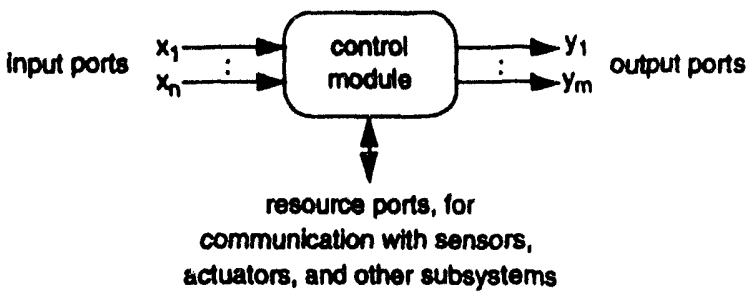

Figure 4. Model of a Port-Based Object (D. B. Stewart et al, ref. 16).

Modules exchange information through a global database of state information (Figure 5); the database is implemented with global-shared memory. Every input/output ( $\mathrm{I} / \mathrm{O})$ port is state variable. A global state variable table that contains all module $\mathrm{I} / \mathrm{O}$ port variables is stored in the shared memory. Tasks 
corresponding to each control module cannot access the table directly; instead, each task has its own local copy of the table. Only the variables used by the task are kept up to date. At the beginning of every task cycle, the variables that are input ports are copied from the shared table. At the end of the task cycle, the output ports are copied to the shared table. The global table is locked whenever a task is transferring data between its table and the global table. The global state variable table mechanism has been incorporated into the Chimera 3.0 Real-Time Operating System. The target architecture is a VME-based multiprocessor system.

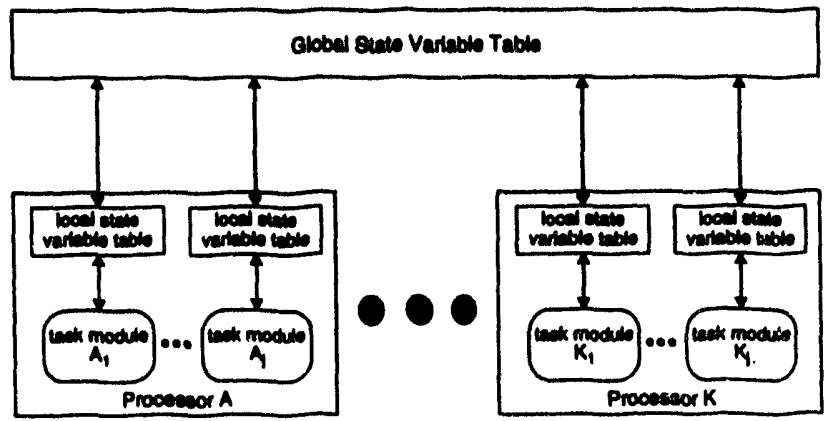

Figure 5. Structure of a State Variable Table Mechanism (D. B. Stewart et al, ref. 16).

\section{Traded and Shared Control}

The NASA-Jet Propulsion Laboratory (JPL) has developed a system that will allow operation from either a teleoperator or an autonomous planner-or a combination of the two. ${ }^{18}$ Thus, the system will have shared as well as traded control. The generation of trajectories can be approached in two ways. First, a nominal autonomous trajectory can be modified by a teleoperator. Second, a nominal teleoperator trajectory may be modified autonomously. Emphasis is placed on controlling a remotely located robot in the presence of transmission time delays. The architecture allows us to start in a completely teleoperated mode and move toward complete autonomy.

\section{GISC}

Sandia National Laboratories, along with the other DOE laboratories, is developing a GISC concept. The DOE's RTDP is sponsoring this program. GISC, a communications-based architecture, follows the premise that sophisticated intelligent system performance is achieved by coordinating a collection of semiautonomous subsystems, each with complementary capabilities (Figure 6). ${ }^{4}$ Each subsystem has a well-defined command-and-control interface, and a supervisory control program coordinates the overall activities of the system through these subsystem interfaces. Individual subsystems may also possess real-time low-level control functions that can be performed autonomously and asynchronously. With the correct combination of supervisor and subsystem capabilities, such an approach supports the implementation of model-based control and sensor integration within reusable software structures. This approach also encourages the use of modularity, distributed multiprocessing environments, and standard commercial interfaces.

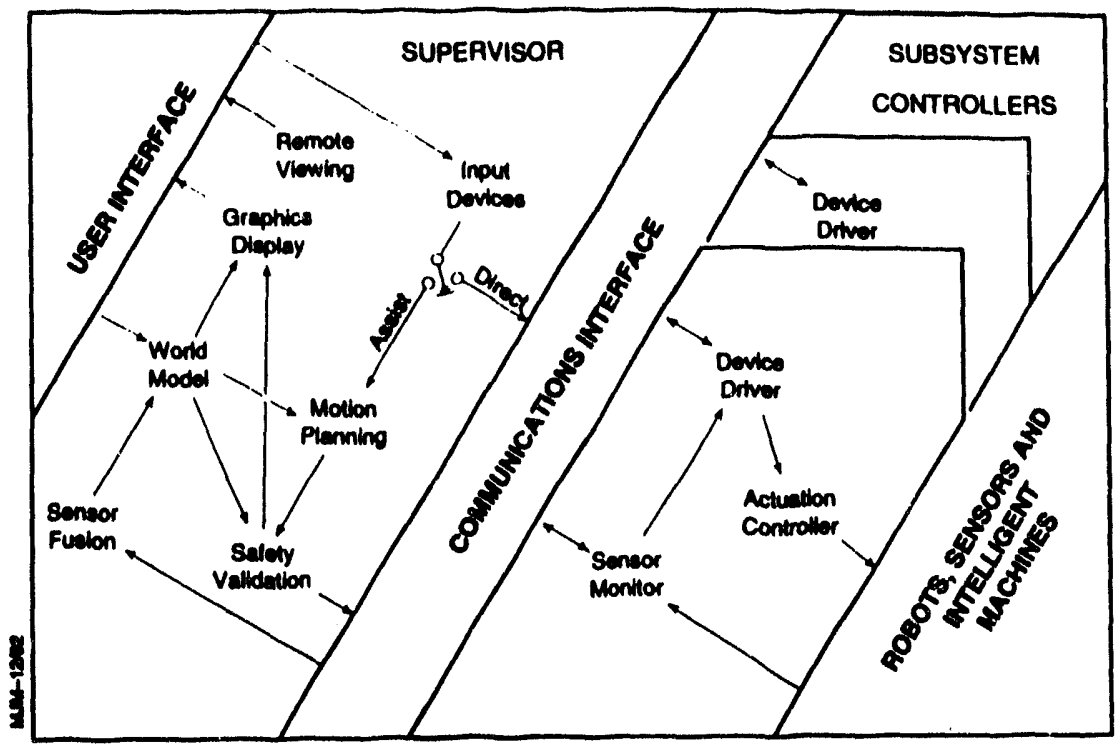

Flgure 6. Generic Intelligent System Controller (GISC) Architecture (M. J. McDonald and R. D. Palmquist, ref. 22). 
To build a GISC-based system, a set of tools is required for developing the supervisor and subsystems and for integrating the pieces into an operational control system. The laboratories have developed five tools that provide a range of capabilities required by an intelligent system. These include:

- The Intelligent System Operating Environment (ISOE), which provides the low-level communication facilities.

- The General Interface for Supervisor and Subsystems (GENISAS), which provides the highlevel communication framework needed for a distributed supervisor/subsystem arrangement (Figure 7). ${ }^{19}$ GENISAS uses the client/server approach to break the sysiem up into separate processes that communicate through messages.

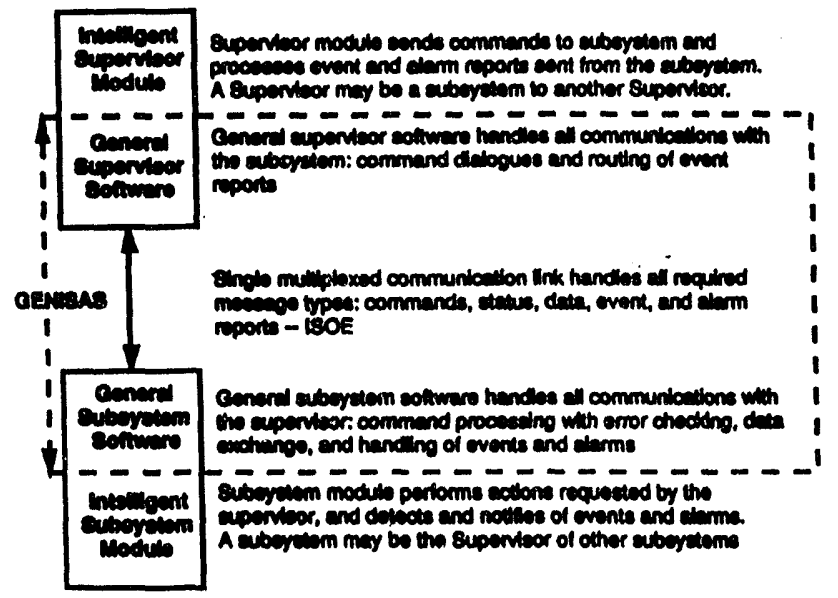

Figure 7. GENISAS Supervisor and Subsystem Interface (J. M. Griesmeyer, ref. 19).

- The Robot Independent Programming Environment and Language (RIPE/RIPL), which enables development of generic subsystems by providing object-oriented interfaces to intelligent system devices. ${ }^{20}$

- The Sequential Modular Architecture for Robotics and Teleoperation (SMART), which provides an underlying stable control system that has the performance and flexibility for sensorbased control and teleoperation, including force reflection. ${ }^{21}$ The SMART component of GISC is also capable of shared and traded control modes with force reflection.

- The Graphical Programming Environment (Sancho), which provides an easily reconfigurable, menu-based, operator interface and an on-line simulation environment. ${ }^{22}$
GISC has been built around the concept of online graphical simulation with subsequent operatorapproved robot motion and supervisory control with a graphical interface that is menu and mouse driven. Many systems have been successfully deployed using the GISC approach. Some examples of these systems include remote hazardous waste cleanup demonstrations, EPA chemical laboratory automation initiatives, large-structure painting, remote vehicle controlling, and agile manufacturing. All of these systems have been implemented on UNIX workstations coupled to VME multiprocessing environments using the VxWorks real-time operating system. In its current form, GISC is a loosely defined specification that allows more detailed interface specifications to be defined for each subsystem as the need arises. An effort is currently underway to develop a detailed generic robot subsystem interface specification. ${ }^{2}$

\section{A Telerobotic Architecture for Large Aircraft Maintenance}

NASA-JPL has completed a conceptuai design for hazardous operations involving stripping, cleaning, finishing, and painting of large aircraft structures. The concept of the generic telerobotic control architecture focuses on a task sequence coupled with a world model. ${ }^{23}$ Three elements are involved in system operation:

- First, the object-modeling modules provide a model of the hardware and task environments.

- Second, the task-description modules provide a means to build task programs.

- Third, the task-execution modules perform simulations or drive devices.

The hardware architecture is broken up into two locations: local and remote. The software is broken up into modules along functionality lines, which provide a means for easy system development, modification, and upgrades. For example, the modules include an object knowledge base, object calibration, parent task program sequencer, subsystem task program sequencer, subsystem task level control, robot servo control, and robot (Figure 8). The NASA-JPL report (ref. 23) concludes by recommending areas for additional study, one of which is a tradeoff analysis of the actual command/data types and structures of module communication. The intent is to minimize the amount of internal data translation required. A detailed design of a system using this approach has not been developed. 


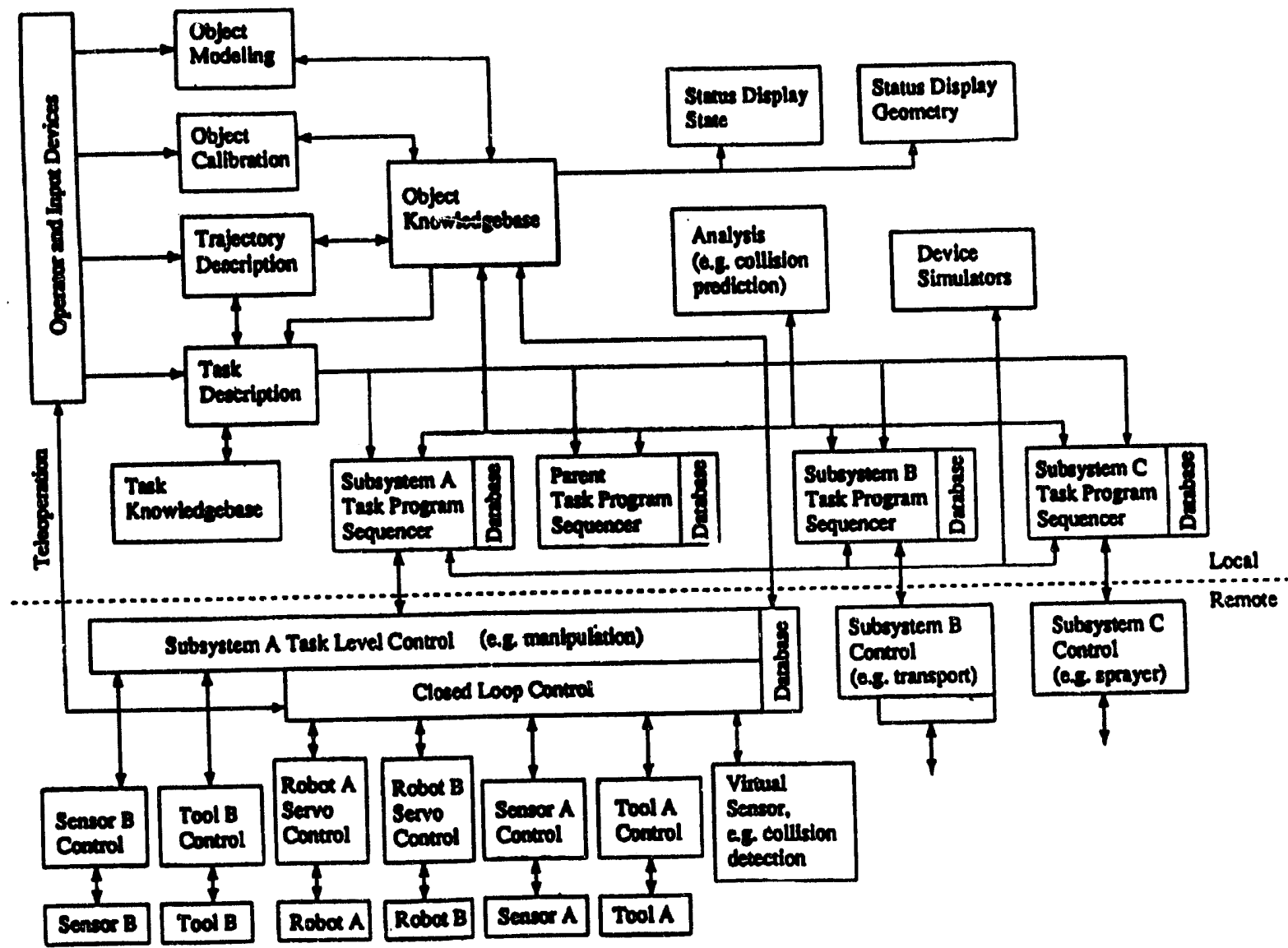

Figure 8. Generic Telerobot Control Architecture (NASA-JPL, ref. 23).

\section{NOMAD}

Trellis Software \& Controls is developing NOMAD, a set of motion-control software modules that can be combined with off-the-shelf computer hardware and software to build an open architecture robot or motion controller. ${ }^{24}$ The base operating system for the product is LynxOS ${ }^{\mathrm{TM}}$, a POSIX-compliant realtime version of UNIX. NOMAD and LynXOS ${ }^{\text {TM }}$ run on PC-ATs and VME-based processors. The system is programmable in C, with full UNIX compatibility, including X-Windows, Motif, NFS, and TCP/IP networking. A controller comprised of NOMAD and off-the-shelf computer components provides the full power of sensor-controlled robotic motion with userreplaceable kinematics. The current configuration contains three components:

- A trajectory generator, which provides sensorcontrolled motion and a variety of industrial servo and $\mathrm{I} / \mathrm{O}$ interfaces

- A user environment, which consists of a C library (for communicating with the trajectory generator) and system configuration tools and example programs

- Several utilities such as the graphical servotuning tool, machine simulator, command line interface, and teach pendant support. Trellis Software and Controls is currently developing a robot language and a numerically controlled environment for NOMAD.

\section{ROBLINE}

CIMETRIX, Inc., is developing an open architecture controller capable of controlling a diverse array of machines under a standards-based operating environment and built on industry-standard hardware platforms. ${ }^{25}$ This system also uses the Lynx $\mathrm{OS}^{\mathrm{TM}}$. In addition to a main processor (Motorola or Intel), each controller contains at least one Motorola DSP56001 digital signal processor for low-level servo control and I/O monitoring. The controller uses a client/server model to isolate the application programming environment from the low-level control functions. Client/ server programming breaks the control system up 
into separate processes that communicate through messages. The server consists of multithreaded processes for task sequencing, motion planning, and I/O. The server maintains a knowledge base for world models, control parameters, and other information. The clients can be other control system components, a general tool, or an application program. For off-line programming and simulation, the servo subsystem can be replaced with a graphics subsystem.

A C library of utility functions is provided to the applications programmer. The library contains functions for motion control, I/O and process control, geometric modeling, user interface functions, and many others. Also included is the Non-Programmers Interface, which is a C-interpreter, computer-aided, software engineering tool that allows users to select functions from an $\mathrm{X}$-Windows program.

\section{Adept}

The NASA-JPL final report, which focused on commercial technologies for hazardous operations involving large aircraft structures, surveyed the marketplace in 1993 for robot controllers. ${ }^{23}$ Their report included the information as summarized above for the NOMAD and ROBLINE controllers. However, a third offering is the AdeptMotion Servo controller. The ADEPT architecture allows developing and tailoring custom software modules within the primary controller to drive the various device actuators.

\section{Hardware}

This report does not cover in detail the standard hardware architectures for robot controllers, because most robot controllers use proprietary designs. Only recently has the robotics industry begun to respond to repeated requests from the robotics $R \& D$ community for open architectures. In 1991 a backplane bus study geared towards the area of space automation and robotics compared four different bus architectures. ${ }^{26}$ Category scores were weighted based on their particular application arena. The categories included technical capability, reliability, commercial support, military support, module cost, module availability, and risk. The scoring criteria for each category were fully documented. The study recommended the VME bus for current use and Futurebus + for long-term selection as a standard. Futurebus + is a new standard chosen by the U.S. Navy and will not be fielded in any systems until 1996. Futurebus + is an extensible, fast backplane that will allow any processor from 32 bits to 128 bits to occupy a slot in the chassis. The high cost, low availability, and poor support will hinder research activities; however, Futurebus + will become increasingly more attractive after the Navy fields several systems. The performance and support for advanced applications will demand an alternative to the VME bus in a few years.

\section{Past Specifications Related to this Procurement}

Thill section describes two former specifications related to the procurement of the LRM robotic system.

\section{Extended Surface Manipulator}

The Extended Surface Manipulator is a 32-foot, 11-degree-of-freedom hydraulic robot. This robot is a custom arm with a hybrid control system. The GISC approach was used to provide all of the high-level control capability down to the level of individual servo control. Schilling Robotics, the manufacturer of the arm, provided the low-level element of servoing to joint positions. The GISC approach, through the use of SMART, computes the trajectory and feeds the joint angles tc a VME/VxWorks interface at a rate of $50 \mathrm{~Hz}$. The low-level controller servos to these set points at the same $50-\mathrm{Hz}$ rate, thus resulting in the desired smooth motion.

The other interfaces between the GISC approach and Schilling Robotics' low-level control included startup and initialization commands (e.g., turning the hydraulic power unit on), various error-tracking capabilities (e.g., the difference between commanded and actual positions), and error-handling routines (e.g., emergency stops).

\section{LDUA}

The Light-Duty Utility Arm (LDUA) project administered by Westinghouse Hanford Company (WHC) has received a conceptual design report that includes a GISC compatibility statement. ${ }^{27}$ The LDUA control subsystem will be a VME bus-based hybrid control architecture running the VxWorks real-time operating system. All VME boards will be standard off-the-shelf items. The arm and its control system will be based on an existing Spar Aerospace Limited design. All controller software will be implemented with the $\mathrm{C}$ language. The design will include a GISC-based interface to the LDUA control subsystem through C library functions supplied by Spar. The subroutines will be part of the following functionality groups: 
- Motion-simulated or actual, and relative or absolute, for different coordinate systems, with the ability to specify position and rate.

- Kinematics-forward and inverse.

- Context-ability to change system parameters sich as coordinate systems, default rate and acceleration profiles, servo parameters, sensor characterie ics, and physical units.

- Diagnostics-self-testing.

WHC plans to add specific GISC-compatible C library routines to the robot control system specification. A fow representative examples are provided in the appendix.

\section{Conclusions and Recommendations}

With the vast amount of hardware and software for the control of robotic systems and the rapid evolution of these systems, trying to standardize on a specific controller architecture will be difficult. A preferred approach is to use an incremental method by which a common, yet adaptable, architecture could be realized. ${ }^{2}$ The first step is to address standard requirements for individual technologies and the interfaces required to make them work together effectively. One method, which has proven effective in many areas ranging from computer networking to robotic systems, is a modular communications-based philosophy that provides building blocks for intelligent systems. As the number and type of modules increase and as they are made more robust and easier to use, de facto standards may evolve that are based on these common interfaces. This evolution will also ensure adaptability, and a market-driven standardization similar to the IBM PC could result. This process will take several years to mature because of competitive and rapidly evolving technologies. Until that time, the use of VME systems running either VxWorks or LynxOS ${ }^{\mathrm{TM}}$ is the best choice.

If we compare the architectures reviewed in this report, we conclude that they are overly restrictive, because they define individual module functionality. In other words, a vendor or a system integrator does not have much flexibility to develop new or different modules as the need arises. A better method is to define the means of communication and the module interfaces, and let the system integrator choose or invent modules to fulfill the required functionality. With this method, the architecture would not become obsolete overnight because of technology improve- ments or new functionality required of the system. This leads to a communications-based philosophy such as the one used in the GISC approach or the CMU port-based object approach. Other control approaches that are similar to the above approaches are promising; however, because they are in the early stages of development, they have not been tested in an advanced control architecture with multiple robots and multiple sensor systems. Some of these approaches have been abandoned. R. W. Harrigan enumerated several of the best reasons for using a communications-based approach: ${ }^{28}$

- "...Demonstrated that it works...." A communications-based approach has been successful in several demonstrations at WHC and other DOE facilities.

- "...In-depth friendly expertise available...." Software has always been the most difficult portion of a robot system to develop and debug. A large resource of world-class DOE and university experts in the communications-based approach can maintain and develop new control functionality.

- "...Expertise moving to Hanford...." Considerable effort has been expended to generate resident communications-based expertise at WHC, and we should not throw away this resource.

- "...Expertise available throughout the DOE...." The Savannah River Technology Center, $\mathrm{Pa}$ cific Northwest Laboratory, and Oak Ridge, Los Alamos, and Sandia National Laboratories can help WHC.

- “...A communications-based approach supports technology insertion...." For example, the GISC approach was specifically developed to allow modularity. Several hardware and software subsystems have been integrated into existing demonstrations for the Underground Storage Tank (UST) platform (e.g., a GISC-based control system, commercial graphics software, two diverse commercial robots (Spar 2500 and Titan II), a water cannon, computer-guided pipe cutters, and a sludge-removal system). It all worked even though each subsystem was developed separately.

- “...A communications-based approach is widely used...." Decontamination and Decommissioning (D\&D), Work for Others (WFO), Waste Processing Operations (WPO), UST, and Contaminant Automated Analysis (CAA) are using the communications-based approach, and the wealth of experience is growing. 
Another reason to use the above components is the LDUA project. ${ }^{27}$ The LDUA will be using a communications-based approach; therefore, to reduce training requirements, support personnel, and maintenance, a similar architecture on subsequent systems is extremely cost effective. Another project that will use a communications-based approach for control is the RTDP Tank Waste Retrieval test bed. ${ }^{29}$ The test bed supports project W-340 and other arm-based retrieval projects in the DOE. Having different control approaches on the test bed and the fielded robot system may severely hamper the test bed development effort's impact on the actual retrieval systems to be used in W-340. Having two related offices in the DOE that work closely on a project, using the same system control architecture, makes sense and fosters rapid technology transfer as a team environment. Also, compatibility with a communications-based approach is being increasingly specified by DOE in its robot procurements, and other organizations are adoptirig this specification.

Procurement documents must be written, and for the evaluation board to adequately evaluate and grade the vendors, we need to write the documents so that responding vendors adequately detail all of the important aspects of the project Therefore, we should minimize the latitude of control system architectures that a vendor can use in a project, especially when internal expertise has been developed and this project relates to other WHC and DOE RTDP programs. As previously discussed, NIST, the DOE, and others have adopted and developed control architectures around the VME bus. The VME bus provides a flexible and cost-effective multiprocessing, real-time computer hardware/software environment. Futurebus + may soon begin to replace VME, but it will probably be many years before a robust supply of hardware and software exists.

In summary, an effective open architecture should offer the following:

- A multiprocessing environment that is scalable (e.g., VME-bus).

- A multitasking/multithreaded, real-time operating system (e.g., VxWorks).

- A system software code that is written with a data-abstraction-capable language with objectoriented features (e.g., $\mathrm{C}++$ ) structured in a modular fashion. The software should also have the requirement of real-time operation.

- Standardized interfaces rather than subsystems (e.g., GISC). A communications-based philosophy should be required so that interfaces to the subsystems offer easily callable and well- behaved subroutines or functions (e.g., $\mathrm{C}$ or $\mathrm{C}++$ subroutines) with the source code delivered to the buyer. (Refer to the appendix for examples of proposed LDUA project specifications.)

- Adequate resources for maintaining the existing code and for developing new functionality.

\section{References}

${ }^{1}$ R. W. Harrigan, "Automating the Operation of Robots in Hazardous Environments," IEEE/RSJ International Conference on Intelligent Robots and Systems, Yokohama, Japan, July 26-30, 1993.

${ }^{2}$ D. J. Miller, "Standards and Guidelines for Intelligent Robotic Architectures," Proc of AIAA Space Programs and Technologies Conference and Exhibit, September 21-23, 1993.

${ }^{3} E$. Red and M. Dixon, "Survey of Robotic Enabling Technologies for WINCO/DOE," study sponsored by WINCO/DOE and conducted for Idaho Nuclear Engineering Laboratories (INEL) under contract C85-110766 (HRS200-90), September 1990.

'J M. Griesmeyer, M. J. McDonald, R. W. Harrigan, P. L. Butler, and B. Rigdon, "Generic Intelligent System Controller (GISC)," SAND92-2159, Sandia National Laboratories, Albuquerque, NM, October 1992.

${ }^{5}$ R. Quintero, ed., "DOE/NIST Workshop on Common Architectures for Robotic Systems," NIST Special Publication 784, April 1990.

${ }^{6} \mathrm{R}$. Quintero, ed., "Proceedings of the Second DOE/ NIST Workshop on Common Architectures for Robotic Systems," Seattle, WA, January 23-24, 1991, available from NIST, Robot Systems Division, Building 220, Room B124, Gaithersburg, MD 20899.

${ }^{7}$ Department of Energy Office of Environmental Restoration and Waste Management Office of Technology Development, "System Controller Project Review for the Robotics Technology Development Program," Oak Ridge National Laboratory (ORNL), November 17-19, 1992.

J. S. Albus, H. G. McCain, and R. Lumia, "NASA/ NBS Standard Reference Model for Telerobot Control System Architecture (NASREM)," NIST Technical Note 1235, April 1989.

${ }^{9}$ Martin Marietta, "Draft Volume I of Next Generation Workstation/Machine Controller (NGC) Specification for an Open System Architecture Standard (SOSAS)," Document No. NGC-0001-13-000-SYS, March 1992.

${ }^{10} \mathrm{M}$. B. Leahy and S. B. Petroski, "Unified Telerobotic Architecture Project Program Overview," submitted to the 1994 IEEE International Conference on Intelligent Robots and Systems (IROS).

${ }^{11}$ F. Hayes-Roth, L. D. Erman, A. Terry, and B. HayesRoth, "Domain-Specific Software Architectures: Distributed Intelligent Control and Communication," IEEE Symposium on Computer-Aided Control System Design, pp. 117-128, Napa, CA, March 1992. 
12F. Hayes-Roth, L. D. Erman, R. London, and A. Terry, "Tools and Methods for Developing Distributed Intelligent Control and Management (DICAM) Application Systems," position paper for the Joint Services Guidance and Control Committee (JSGCC) Software Initiative Workshop, October 13, 1992.

${ }^{19}$ G. N. Saridis, "Architectures for Intelligent Controls," IEEE Symposium on Implicit and Nonlinear Systems, Ft. Worth, TX, December 14-15, 1992.

${ }^{14}$ A. Elfving and U. Kirchhoff, "Design Methodology for Space Automation and Robotic Systems," ESA Journal, Vol. 15, 1991.

${ }^{16}$ T. M. Mitchell, "Becoming Increasingly Reactive," AAAI-90 Proceedings: Eighth National Conference on Artificial Inte!ligence, Vol. 2, pp. 1051-1058, July 29-August 3, 1990.

${ }^{16}$ D. B. Stewart, R. A. Volpe, and P. K. Khosla, "Integration of Real-Time Software Modules for Reconfigurable Sensor-Based Control Systems," Proceedings: 1992 IEEE/ RSJ International Conference on Intelligent Robots and Systems, Psleigh, NC, July 7-10, 1992.

${ }^{17}$ D. B. Stewart, R. A. Volpe, and P. K. Knosla, "Design of Dynamically Reconfigurable Real-Time Software using Port-Based Objects," submitted to IEEE Transactions on Software Engineering, July 1, 1993.

${ }^{18}$ S. Hayati and S. T. Venkataraman, "Design and Implementation of a Robot Control System with Traded and Shared Control Capability," Proceedings: 1989 IEEE International Conference on Robotics and Automation, Vol. 3, pp. 1310-1315, Scottsdale, AZ, May 14-19, 1989.

${ }^{19} \mathrm{~J}$. M. Griesmeyer, "General Interface for Supervisor and Subsystems (GENISAS)," First Review Draft, Sandia National Laboratories, Albuquerque, NM, October 1992.

${ }^{20}$ D. J. Miller and R. C. Lennox, "An Object-Oriented Environment for Robot System Architectures," IEEE Control Systems, Vol. 11, No. 2, February 1991.
${ }^{21}$ R. J. Anderson, "SMART: A Modular Architecture for Robotics and Teleoperation," Proceedings: IEEE International Conference on Robotics and Automation, Atlanta, GA, May 26, 1993.

${ }^{22}$ M. J. McDonald and R. D. Palmquist, "Graphical Programming: On-line Robot Simulation for Telerobotic Control," Proceedings of the International Robots and Vision Automation Conference, Detroit, MI, April 5-8, 1993.

${ }^{23}$ NASA-JPL, "A Generic Telerobotics Architecture for C.5 Industrial Processes: Final Report," prepared for Air Force Material Command (AFMC) Robotics and Automation Center of Excellence (RACE), San Antonio Air Logistics Center, Kelly AFB, TX, 78241, August 1993.

${ }^{24}$ L. Rossol, "Nomad Open Architecture Motion Control Software," Proceedings of the International Robots and Vision Automation Conference, Detroit, MI, April 5-8, 1993.

${ }^{26} \mathrm{~S}$. Sorensen, "Overview of a Modular, Industry Standards based Open Architecture Machine Controller," Proceedings of the International Robots and Vision Automation Conference, Detroit, MI, April 5-8, 1993.

${ }^{26}$ G. Szatkowski, "Backplane Bus Trade Study," submitted to AIAA Committee on Standards for Space Automation \& Robotics, April 29, 1991.

${ }^{27}$ SPAR, "Light Duty Utility Arm (LDUA) and Deployment System: Conceptual Design Report," unpublished report, under contract to Westinghouse Hanford Company, Richland, WA 99352, October 1993.

${ }^{28} \mathrm{R}$. W. Harrigan, "Why UST Should Use GISCUpdate," memo to Dan Horschel, Org. 1661, Sandia National Laboratories, Albuquerque, NM, October 4, 1992.

${ }^{29}$ Tank Waste Retrieval Test Manipulator Project, "Technical Specifications: Tank Waste Retrieval Test Manipulator System," unpublished, Pacific Northwest Laboratory (PNL), September 30, 1993. 


\section{APPENDIX}

\section{Proposed C Functions}

This appendix describes some of the proposed C functions that will allow the communications-based, GISC-compatible, supervisory control system of the LDUA to communicate with the SPAR robot controller. Crary Kiebel and Edward Allis of Westinghouse Hanford Company (WHC) are the authors, and William E. Ford edited the text.

\section{General Functions}

\section{IduaLock()}

A function that places the subsystem controller into a state where it will accept commands only from the GISC interface, and not accept commands from any of its control consoles or panels, so that the LDUA cannot be operated from them while under GISC control. This function shall properly coordinate with the point-of-control feature of the control system; for example, it shall fail if point-of-control is currently locked at a local control panel.

\section{IduaRelease()}

A function that will reverse the effect of IduaLock().

\section{IduaInit()}

An initialization function that will set up the proper software environment for all the $\mathrm{C}$ functions.

\section{Arm Control Functions}

\section{IduaMoveByJoint(jt1, jt2, ..., jt8)}

A function that causes the LDUA mast and arm to move to a desired point defined in joint space. The function shall return immediately and not wait for the move to be completed. This function shall not move the mast and arm and shall return an error code if GISC is not currently the active point-of-control (see IduaLock()).

IduaMoveByCartesian(x, y, z, roll, pitch, yaw) A function that causes the LDUA mast and arm to move to a desired point defined in cartesian space.
The function shall return immediately and not wait for the move to be completed. This function shall not move the mast and arm and shall return an error code if GISC is not currently the active point-of-control (see IduaLock()).

IduaMoveByToolSpace( $x, y, z$, roll, pitch, yaw)

A function that causes the LDUA mast and arm to move to a desired point defined in tool space. The function shall return immediately and not wait for the move to be completed. This function shall not move the mast and arm and shall return an error code if GISC is not currently the active point-of-control (see IduaLock()).

\section{IduaMoveByJointPath(setOfJointPosition Sets)}

A function that causes the LDUA mast and arm to move in a path defined as a set of goal points (jt1, jt2, ...jt8) in joint space. The function shall return immediately and not wait for the move to be completed. This function shall not move the mast and arm and shall return an error code if GISC is not currently the active point-of-control (see IduaLock()).

\section{IduaMoveByCartesianPath(setOfGoalPoints)}

A function that causes the LDUA mast and arm to move in a path defined as a set of goal points $(x, y$, $z$, roll, pitch, yaw) in cartesian space. The function shall return immediately and not wait for the move to be completed. This function shall not move the mast and arm and shall return an error code if GISC is not currently the active point-of-control (see IduaLock()).

\section{IduaMoveByToolPath(setOfGoalPoints)}

A function that causes the LDUA mast and arm to move in a path defined as a set of goal points $(x, y$, $\mathrm{z}$, roll, pitch, yaw) in tool space. The function shall return immediately and not wait for the move to be completed. This function shall not move the mast and arm and shall return an error code if GISC is 
not currently the active point-of-control (see IduaLock()).

\section{IduaEnableDrives(enable/disable)}

A function that invokes the subsystem controller's drive enable/disable feature (an I/O channel may be necessary to interface with the drive enable/ disable hardware). If disabled, the mast and arm drives are prevented from moving. If enabled, they are allowed to move. This function shall return an error code if GISC is not currently the active point-ofcontrol (see lduaLock()).

\section{IduaEnableSimulation(enable/disable. gavePreview:T/F, TIMESTEP or DISTANCE STEP)}

A function that places the subsystem controller into the simulation mode. In this mode, it will accept motion commands as usual, but will not move the mast and aim. It will instead simulate the actual sequence of joint motions in time so that the subsystem controller will drive the arm through to reach the defined goal. The simulated positions will be available on demand for the duration of the simulated move via lduaGetPosition, and will constitute an accurate preview of the motion. The LDUA Supervisory Control System will use the preview for path planning and collision-avoidance checking; therefore, it must be an exact representation of what the subsystem controller will do (it must include the effects of all compensation routines and automatic joint reconfiguration caused by the subsystem controller's collision-avoidance features, if active). Simulation mode is made active by enable and made inactive by disable. When called with savePreview set to TRUE, the subsystem controller shall retain a detailed copy of the simulated motion (which may be subsequently invoked by lduaMoveByPreview). An error shall be returned if drives are not currently disabled (see lduaEnableDrives). This function shall return an error code if GISC is not currently the active point-of-control (see lduaLock()).

\section{IduaMoveBy Preview (warning/stop)}

A function that causes the LDUA mast and arm to execute the current previewed path (see IduaEnableSimulation) that is stored in the subsystem controller. The function shall return an error if there is no valid preview. The subsystem controller shall invoke an event to the GISC CPU if it must deviate from the previewed path during the execution. If the function is called with the "stop" parameter, the subsystem controller will halt motion in the event of path deviation from preview. The "warning" parameter shall permit the motion to continue. The function shall return immediately and not wait for the move to be completed. This function shall not move the mast and arm and shall return an error code if GISC is not currently the active point-of-control (see lduaLock()).

IduaSetBaseTransform(x,y, z, roll, pitch, yaw)

A function that describes the mapping betwren the tank frame coordinate system and the base frame of the mast/arm. This function shall return an error code if GISC is not currently the active point-ofcontrol (see lduaLock()).

\section{lduaSetRiserAngle(xAngle, y Angle)}

A function that inputs the angle of the riser from plumb. The vendor shall provide and clearly mark two datum nodes on the mast containment housing (or other suitable surfaces) where $x$ Angle and yAngle are to be measured. The riser angle is used by the LDUA subsystem controller to estimate the compensation to apply for static deflection of the mast because of gravity. This function shall return an error code if GISC is not cli.rently the active point-ofcontrol (see lduaLock.()).

\section{lduaSetPayload(weight, distanceToCG)}

A function that enters the weight and center of gravity of the end effector that is currently mounted on the TIP. An error is returned if TIP is empty. This function shall return an error code if GISC is not currently the active point-of-control (see IduaLock()).

\section{IduaGetPayload (weight, distanceToCG)}

A function that returns the weight and center of gravity of the end effector that is currently mounted on the TIP. An error is returned if TIP is empty.

IduaSetToolTransform( $x, y, z$, roll, pitch, yaw) A function that sets the tool frame coordinates for the current end effector. This is expressed as a transformation from the TIP frame to the tool frame. An error is returned if TIP is empty. This function shall return an error code if GISC is not currently the active point-of-control (see lduaLock()).

IduaGetToolTransform ( $x, y, z$, roll, pitch, yaw)

A function that returns the transformation from the wrist frame to the tool frame of the end effector that is currently mounted on the TIP. An error is returned if TIP is empty. 
IduaGetParamter(paramName, paramValue, valueType)

A function that reads the value of one set of subsystem controller parameters. The seller shall provide, through this function, access to all the subsystem controller parameters that are not otherwise provided access by the other functions listed here.

\section{IduaSetParamter(paramName, paramValue, valueType)}

A function that sets the value of one of the set of subsystem controller parameters such as maximum tip speed, amplifier power on/off, and trajectory generator cptions. The seller shall provide, through this function, access to all the subsystem controller parameters that are not otherwise provided access by the other functions listed here. These parameters shall include, but not be limited to, maximum tip spaed, trajectory generator options, and input-shaping filter gains. This function shall return an error code if GISC is not currently the active point-of-control (see Idualock()).

\section{IduaTrajectoryPause(pause/resume/cancel)}

A function that causes the current motion of the mast/arm to pause arid resume. When called with pause, the current motion of the mast and arm is brought to a smooth and controlled stop (function returns error if mast and arm are not currently being directed to move), $::$. so functions that change motion parameters will return errors while pause is in effect. When called with resume, the paused motion is resumed (function returiss an error if pause is not in effect). When called with cancel, the current motion command is canceled and the mast and arm remain where they are. If pause is not in effect when call is made, cancel first invokes it. This function shall return an error code if GISC is not currently the active point-of-control (see lduaLock()).

\section{IduaSoftStop()}

A function that invokes the "Soft Stop" feature of the subsystem controller. This function shall return an error code if GISC is not currently the active pointof-control (see IduaLock()).

\section{Arm Status Functions}

IduaGetPosByJoint(jt1, jt2, ..., jt8)

A function that reads the LDUA current position joint space. Under normal mode, the position of the arm is derived from the joint position sensors. Under simulation mode (see IduaEnableSimulation), the position is calculated from the motion planning parts of the system. The position returned under simulation mode is the ideal one. To identify the joint precompensation that the system would apply, to try to achieve the ideal position, the IduaGetJointCompensation function could be used on the ideal joint positions.

IduaGetPosByCartestion(x, y, z, roll, pitch, yaw)

A function that reads the LDUA current position cartesian space. Under simulation mode (see IduaEnableSimulation), the position is calculated from the motion-planning parts of the system. The position returned ur der simulation mode is the ideal one.

IduaGetPosByToolSpace(x, y, z, roll, pitch, yaw)

A function that reads the LDUA current position tool space. Under simulation mode (see IduaEnableSimulation), the position is calculated from the motion-planning parts of the system. The position returned under simulation mode is the ideal one.

\section{lduaSync(Cartesian/Joint)}

A function that will allow the GISC software to sync up to either the joint or cartesian update rates of the LDUA controller. When called, it will block until the next update and then return. The function will return immediately with an error if mast and arm are not currently being directed to move.

\section{IduaMoveComplete()}

A function that will allow the GISC software to determine if the LDUA has completed the movement directed by the most recent IduaMoveBy...() function call.

\section{IduaGetArmStatusItem(itemName, itemValue, valueType)}

A function to read the status of the mast/arm from the subsystem controller. It obtains the value of a single specified status item. The seller shall provide access to all the subsystem controller mast/arm status values through this function or through the IduaGetArmStatusRecord function. Status items shall include health monitoring, emergency stop, and limp mode. 
IduaGetArmstatusRecord (statusCategory, statusRecord)

A function to read the status of the mast/arm from the Subsystem Controller. It obtains the value of a group of related status items specified by category. The seller shall provide access to all the subsystem controller mast/arm status values through this function or through the IduaGetArmStatusItem function.

\section{IduaRunDiagnostic(diagnosticNumber, diagnosticResultRecord)}

A function that provides access to the on-board diagnostic features of the subsystem controller. The seller shall identify the different diagnostics that can be run and the details of the results they return. The function shall return an error if the diagnostic was not successful.

\section{IduaSetEventCallback(eventName, callbackFunction)}

A function whereby the GISC CPU can specify which of its own functions is to be called when a specified asynchronous event occurs in the subsystem controller. An asynchronous event is defined as one that requires the immediate attention of the GISC CPU (as opposed to being polled by the GISC CPU as are all the other functions defined in this section). A typical example would be system faults that do not occur during the execution of one of the other functions (and which could return an error from the function call). The seller shall assure that all the asynchronous events requiring GISC CPU attention are accessible by this function.

\section{Kinematic Model Functions}

IduaForwardKin(Joints, Cartesian Position)

The forward kinematic solution for the LDUA.

IduaInverseKin(Cartesian Position, Joints)

The inverse kinematic solution for the LDUA. An error is returned if no solution exists.
IduaGetJointCompensation(jt1, jt2, ..., jt8)

A function that returns the amount of precompensation that is applied to each joint to correct for the deflections of the mast and arm because of gravity. The function is called with a set of joint positions that define a position of the mast and arm in space. It returns with the amount of compensation that would be applied to each joint when the mast and arm were in that position.

\section{Path-Alteration Functions}

IduaPathAlterationEnable(enable/disable)

A function that enables/disables the Idua PathAlterationUpdate function. The subsystem controller will ignore subsequent updates if called with disable, and will accept them if called with enable. This function shall return an error code if GISC is not currently the active point of control (see IduaLock()).

IduaPathAlterationUpdate(x rr, yErr, zErr, rollErr, pitchErr, yaw Err)

A function that tells the subsystem controller the difference between where the end effector currently is and where it would like to be. The subsystem controller will adjust the current position and trajectory of the mast/arm to try and correct the error. The position error will be expressed in tool space. An error will be returned if path alteration has not been enabled by IduaPathAlterationEnable().

\section{IduaPathAlterationSynch()}

A function that will allow the GISC software to sync up to the update rates of the LDUA controller for the position tracking function. When called, it will block until the next update and then return. The subsystem controller shall be capable of at least a 10-H; update rate. The function shall return immediately with an error if path alteration has not be enabled by lduaPathAlterationEnable(). 


\section{DISTRIBUTION:}

1 Oak Ridge National Laboratories Attn: Bill Hamel

1 Pacific Northwest Laboratories

Attn: Dave Bennett

1 Westinghouse Hanford Co.

Attn: Pete Gibbons

6 Westinghouse Hanford Co.

Attn: Eric Shen

$\begin{array}{rll}1 & \text { MS0322 } & \text { P. J. Eicker, 2100 } \\ 1 & \text { MS0949 } & \text { R. W. Harrigan, 2102 } \\ 1 & \text { MS0951 } & \text { P. A. Erickson, 2103 } \\ 1 & \text { MS0949 } & \text { P. A. Molley, 2111 } \\ 1 & \text { MS0951 } & \text { D. R. Strip, 2121 } \\ 1 & \text { MS0952 } & \text { R. D. Palmquist, 2151 } \\ 1 & \text { MS0952 } & \text { B. R. Davies, 2151 } \\ 24 & \text { MS0952 } & \text { W. E. Ford, 2151 } \\ 1 & \text { MS0952 } & \text { R. J. Anderson, 2151 } \\ 1 & \text { MS0952 } & \text { D. J. Miller, 2151 } \\ 1 & \text { MS0952 } & \text { W. M. Davidson, 2161 } \\ 1 & \text { MS0952 } & \text { W. Griesmeyer, 2161 } \\ 1 & \text { MS0952 } & \text { M. J. McDonald, 2161 } \\ 1 & \text { MS0952 } & \text { D. S. Horschel, 2161 } \\ 1 & \text { MS1006 } & \text { P. Garcia, 2171 } \\ 1 & \text { MS1007 } & \text { A. T. Jones, 2172 } \\ 1 & \text { MS9018 } & \text { Central Technical Files, 8523-2 } \\ 5 & \text { MS0899 } & \text { Technical Library, 7141 } \\ 1 & \text { MS0619 } & \text { Technical Publications, 7151 } \\ 10 & \text { MS0100 } & \text { Document Processing } \\ & & \text { For DOE/OSTI, 7613-2 }\end{array}$



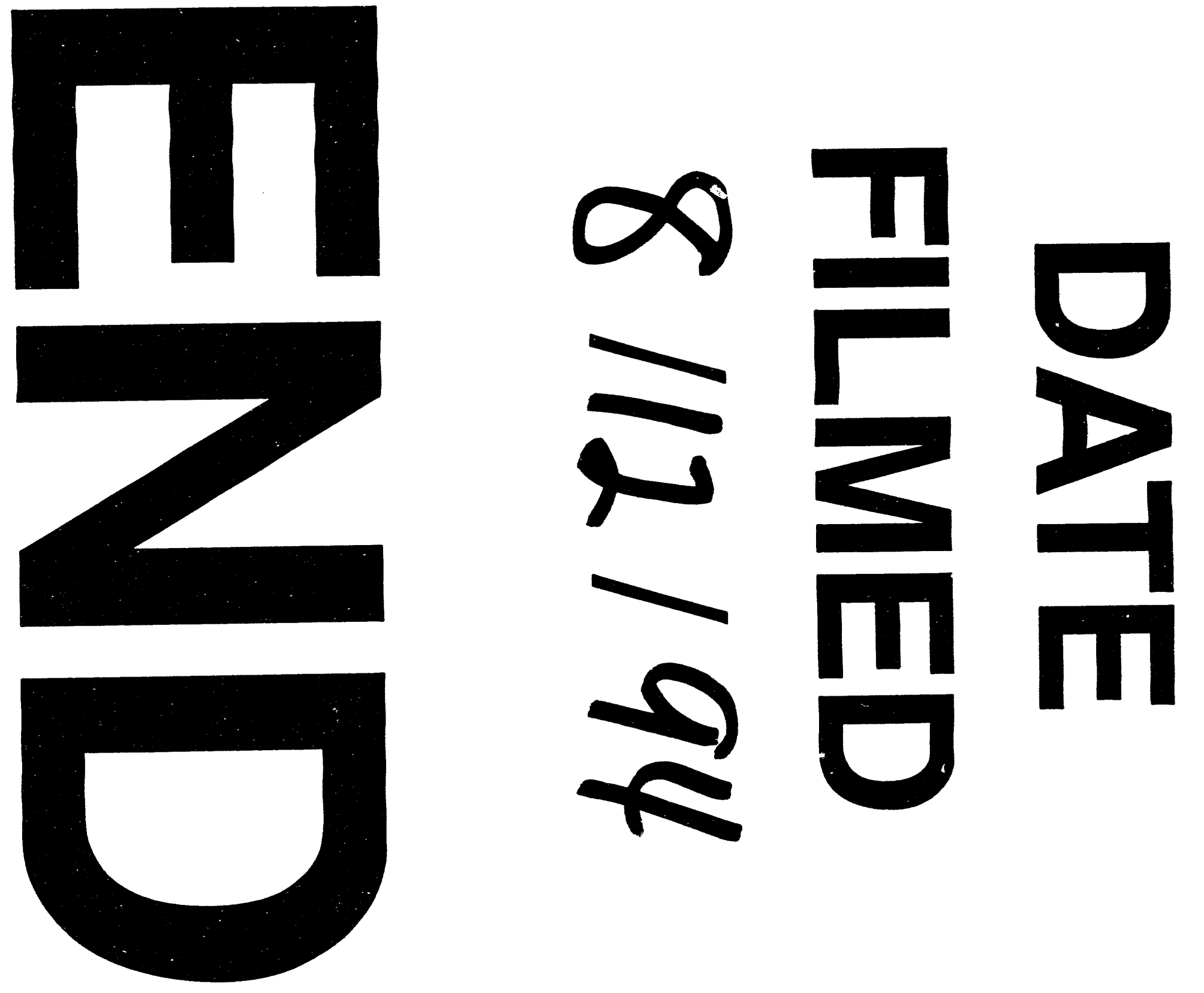


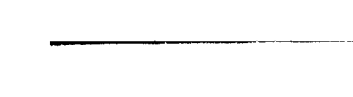

